\title{
Observaciones sobre la cuestión del origen de la lógica en la forma de la trivalencia y la tetravalencia
}

\author{
Por Alexandru Surdu \\ Institutul de Filosofie \\ Academiei Române, Bucarest
}

Desde su primera publicación en 1990, la monografía en la que Niels Öffenberger propone una reconstrucción tetravalente de la lógica asertórica aristotélica ha recibido una amplia atención en la discusión especializada. La obra no sólo fue reseñada y comentada críticamente, sino también traducida a diversas lenguas, incluidas el español y el inglés. A poco de aparecer la obra, yo mismo escribí una amplia reseña crítica que fue publicada en dos versiones: una más amplia en Philosophica (15 [1992] 265-275), y una abreviada (ver Méthexis 6 [1993] 179-189). Podría parecer que un trabajo de este tipo, dedicado a un problema técnico tan específico, muy lejos podría estar de motivar el interés de un público filosófico más amplio, más allá del estrecho círculo de quienes se dedican a la lógica polivalente contemporánea. La repercusión del libro ha mostrado, sin embargo, que esa suposición es equivocada. La razón para este hecho, a primera vista sorprendente, estriba - a mi modo de ver-en el interés estrictamente filosófico, y no sólo meramente histórico o meramente lógico, de los resultados alcanzados en la obra de Öffenberger. Su reconstrucción sistemática de la asertórica 
aristotélica, a partir de las observaciones sobre la cuantificación de los valores de verdad que Aristóteles realiza en su tratamiento de las inferencias a partir de lo falso en Analytica Priora II 2-4, trae consigo un conjunto de consecuencias que impactan de modo directo también sobre el modo de comprender algunas premisas básicas de la epistemología aristotélica. Una vez advertidas esas consecuencias, probablemente no todo el mundo estará dispuesto a pagar los costos que se vinculan con ellas. Pero nadie podrá negar la consistencia interna de la posición presentada por el autor. A lo largo de años, Öffenberger fue puliendo y desarrollando los detalles de su reconstrucción, hasta llegar a un cuadro de conjunto completamente consistente, que (casi) elimina todos los residuos de la presentación tradicional. Para ello, resulta de decisiva importancia la introducción, siguiendo a A. Menne, de los enunciados estrictamente particulares, que permiten no sólo completar el cuadrado de oposición tradicional, sino que incluso obligarían, a mi modo de ver, a una completa reformulación que acarrearía, en último término, la lisa y llana eliminación de los enunciados no estrictamente particulares con los que opera la presentación tradicional, es decir, de los enunciados del tipo I y O. En su nota crítica publicada originalmente en rumano, el profesor Alexandru Surdu, un reconocido especialista, traza un cuadro explicativo que da cuenta del modo en el que se inserta la reconstrucción ofrecida por Öffenberger en el desarrollo de la lógica polivalente contemporánea, que remonta en su origen al sistema trivalente propuesto por Lukasiewicz. La reconstrucción tetravalente de Öffenberger articula intuiciones iniciales que se sitúan en el lado opuesto a las que subyacen al sistema esbozado por el gran lógico polaco. Surdu pone el acento en algunos de los aspectos cruciales que permiten entender las diferencias más importantes que aquí se plantean, a la vez que pone de relieve también los momentos de continuidad en el desarrollo de la problemática discutida. 


\section{Alejandro Vigo \\ Departamento de Filosofía Instituto Cultura y Sociedad (ICS) \\ Universidad de Navarra}

Que la lógica polivalente tiene una prehistoria que duró 2255 años es una quaestio facti. En efecto, si se pone el «big bang» del descubrimiento de la lógica en el año 225 a. C., es decir, en el año del comienzo de la actividad creadora de Aristóteles, y se hace coincidir el descubrimiento «oficial» de la lógica polivalente con 1920, el año de aparición del artículo de Łukasiewicz «Über die dreiwertige Logik» («Sobre la lógica trivalente» $)^{1}$, entonces se puede calcular que la prehistoria de la lógica polivalente ha durado $335+1920=2255$ años. ¿Por qué una prehistoria tan larga? Para tratar la cuestión nos referiremos a la monografía de N. Öffenberger, titulada Sobre la prehistoria de la lógica polivalente, esp. p. 152-159.

En la citada monografía Öffenberger sospecha que hay dos causas fundamentales de la larga prehistoria de la lógica polivalente, a saber:

1. El tercer valor de verdad es un valor de verdad contraintuitivo, que presupone la equivalencia de enunciados opuestos contradictoriamente, y que impide, en

Ver Łukasiewicz 1920; 108. 
razón de su estatuto de caso límite, la referencia a la tetravalencia;

2. La diferenciación aristotélica de verdad y falsedad no fue reconocida como fuente de inspiración de la diferencia entre verdades básicas y derivadas.

Ad 1:

El estatuto de caso límite de la trivalencia concentró las investigaciones, en efecto, sobre el descubrimiento de un tertium non datur. En la bivalencia, la tabla de verdad de la negación excluye, por cierto, la posibilidad de asignar el mismo valor de verdad a enunciados opuestos contradictoriamente: si un enunciado es verdadero, entonces su negación es falsa, y viceversa.

En la bivalencia se excluye un supuesto tercer caso: es imposible, non datur, puesto que tal tercer caso significaría la verdad o falsedad simultánea, es decir, la equivalencia de enunciados opuestos contradictoriamente. El tercer valor de verdad sólo sería posible si enunciados opuestos contradictoriamente pudieran ser portadores del mismo valor de verdad. Pero ello significaría que enunciados opuestos contradictoriamente, los cuales se hallan en relación de contravalencia, podrían ser equivalentes, vale decir: el tercer valor de verdad presupondría una coincidentia oppositorum entre la contravalencia y la equivalencia. Si se admitiera la equivalencia de enunciados opuestos contradictoriamente, entonces tendríamos la siguiente tabla de verdad de la negación: 


\begin{tabular}{c|cl}
$\mathrm{p}$ & $\mathrm{No} \mathrm{p}$ & \\
\hline $\mathrm{V}$ & $\mathrm{F}$ & contravalencia \\
$\mathrm{F}$ & $\mathrm{V}$ & contravalencia \\
$\mathrm{V}$ & $\mathrm{V}$ & equivalencia
\end{tabular}

\begin{tabular}{cc|cl} 
o bien & $\mathrm{p}$ & No $\mathrm{p}$ & \\
\hline $\mathrm{W}$ & $\mathrm{F}$ & $\begin{array}{l}\text { contravalencia } \\
\text { contravalencia } \\
\text { equivalencia }\end{array}$
\end{tabular}

Para descubrir el tercer valor de verdad, hubo que admitir, por tanto, la coexistencia de dos operadores opuestos entre sí, esto es, la contravalencia y la equivalencia.

Dejemos hablar ahora a Łukasiewicz, para comentar luego la interpretación del autor:

Puedo suponer sin contradicción que mi presencia en Varsovia, en un determinado momento del año que viene, por ejemplo, a mediodía del 21 de diciembre, hoy mismo no está decidida, ni en sentido positivo ni en sentido negativo. Por tanto, es posible, aunque no necesario, que en dicho momento yo esté en Varsovia. Bajo tal presuposición, el enunciado 'a mediodía del 21 de diciembre del año que viene estaré en Varsovia' no puede ser hoy ni verdadero ni falso. Pues, si fuera hoy verdadero, mi futura presencia en Varsovia tendría que ser necesaria, lo que contradice la presuposición inicial; y si fuera hoy falso, entonces mi presencia futura en Varsovia tendría que ser imposible, lo que también contradice la presuposición inicial. Por tanto, el mencionado enunciado no es hoy ni verdadero ni falso y debe tener un tercer valor de verdad, diferente de ' 0 ', es decir, 'falso', y de ' 1 ', es decir, verdadero. Podemos designar dicho valor de verdad con $1 / 12$ '. Se trata, en efecto, de 'lo posible', que aparece como tercer valor de verdad, junto a lo verdadero y lo falso. A este pensamiento debe su origen el sistema trivalente del cálculo proposicional. Se trataba, pues, de indicar la matriz sobre la base de la cual se pudiera definir el nuevo sistema de lógica. De inmediato se me hizo claro que si el enunciado concerniente a mi presencia en Varsovia po- 
see el valor $1 / 2$, entonces su negación debe poseer el mismo valor $1 / 2$. Por tanto, obtuve la equivalencia: $\mathrm{N}^{1} / 2=1 / 2^{2}$.

La matriz sería, pues, la siguiente:

\begin{tabular}{c|c}
$\mathrm{p}$ & No $\mathrm{p}$ \\
\hline 1 & 0 \\
$1 / 2$ & $1 / 2$ \\
0 & 1
\end{tabular}

Quisiéramos señalar lo siguiente: si Łukasiewicz hubiera afirmado «es posible que yo esté presente en Varsovia a mediodía del 21 de diciembre del año que viene» o bien «es posible que yo no esté presente en Varsovia a mediodía del 21 de diciembre del año que viene», entonces habría tenido dos enunciados con el mismo operador de posibilidad, cuyos dicta se oponen contradictoriamente, y ambos enunciados serían verdaderos: 'es posible $\mathrm{p}$ ' y 'es posible $\mathrm{Np}^{\prime}$. Pero la verdad no es un tercer valor de verdad. En cambio, si se deja de lado el factor modal de los dos enunciados mencionados, entonces aparecen dos enunciados asertóricos que se oponen contradictoriamente, pero que, por su sentido, pueden tener ambos el mismo valor de verdad. Ahora bien, un valor de verdad que no excluye su asignación simultánea a enunciados contradictorios, no puede ser idéntico ni con la verdad ni con la falsedad. De ahí que el autor sospeche que el descubrimiento del tercer valor de verdad tuvo lugar porque Łukasiewicz transformó el «modus» del operador modal de la posibilidad en un valor de verdad del dictum asertórico

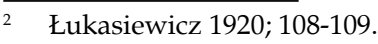


del correspondiente enunciado modal compuesto, dejando de lado, de modo tácito, el factor modal, el modo del enunciado.

Tendríamos, pues, una tabla de verdad de dos enunciados modales, con el operador modal de la posibilidad $(=\mathrm{M})$ y con dicta contradictorios

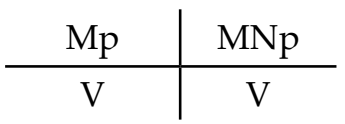

Mediante la eliminación del operador modal, tenemos una tabla de verdad con dicta contradictorios, a saber:

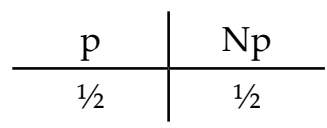

donde ' $\mathrm{p}$ ' es un enunciado que no es ni verdadero ni falso, puesto que, por su sentido, da expresión a los contingentia futura.

Que Łukasiewicz descubrió el tercer valor de verdad al dejar de lado el operador modal de un enunciado verdadero, dando lugar así a un enunciado asertórico que no es ni verdadero ni falso, por no tener correlato objetivo, es una suposición del autor, que no está probada, pero resulta asumible. ¿O más bien ocurrió que Łukasiewicz complementó el ámbito de valor de los enunciados asertóricos que sólo son verdaderos o falsos por medio de los enunciados que no tienen correlato objetivo, puesto que contienen, desde el punto de vista del sentido, eventos futuros contingentes, y que, por lo mismo, son portadores de un tercer valor de verdad?

Los dos caminos de descubrimiento del tercer valor de verdad -sea a través de la eliminación del operador modal de enunciados modales verdaderos con dicta contradictorios, sea a través de la consideración de enunciados opuestos contradictoriamente que, por su sentido, no poseen correlato objetivo- presuponen el funcionamiento simultáneo de ope- 
radores opuestos contradictoriamente, la contravalencia y la equivalencia, en la tabla de verdad trivalente de la negación. Que la tabla de verdad trivalente de la negación necesita la función de operadores opuestos -la contravalencia en la primera y tercera línea, y la equivalencia en la segunda- es, en opinión del autor, en razón de su contraintuitividad, la causa principal del tardío descubrimiento de la lógica polivalente, en la forma de la trivalencia.

No comentaremos otras reflexiones del autor [referidas a los factores] que habrían impedido [un desarrollo más temprano de] la polivalencia en la forma de la trivalencia, a saber: el estatuto liminar, mínimo, del tercer valor de verdad, que habría impedido la investigación en dirección de la tetravalencia (la tetravalencia no supone el funcionamiento simultáneo de equivalencia y contravalencia), y la identidad sintáctica de los enunciados que son portadores del tercer valor de verdad (son idénticos en su forma enunciativa a los enunciados bivalentes verdadero y falsos: 'S es $\mathrm{p}^{\prime} \mathrm{y}$ 'S es No $\mathrm{p}^{\prime}$ pueden ser portadores de cada uno de los tres valores de verdad), que habría dificultado la eliminación del tercer valor de verdad. Con todo, se puede mencionar el hecho de que el ámbito de valor correspondiente a los enunciados que son portadores del tercer valor de verdad tiene una amplitud menor que el de los enunciados verdaderos y falsos, puesto que se trata exclusivamente de enunciados que, por su sentido, no tienen correlato objetivo.

Por otro lado, el autor observa que el descubrimiento del tercer valor de verdad no tuvo como presupuesto ningún contexto científico específico ${ }^{3}$-por caso, el cálculo infinitesi-

\footnotetext{
Łukasiewicz ha observado al pasar tan sólo lo siguiente: «ein System der dreiwertigen Logik, dessen Umrisse ich 1920 angeben konnte, unterscheidet sich von der zweiwertigen
} 
mal, el dodecafonismo o la geometría no euclidiana-. Para justificar la tercera línea de la tabla de verdad trivalente de la negación, no se necesita ningún algoritmo complejo, a pesar de que dicha tabla contiene una coincidentia oppositorum, la acción de operadores opuestos en la misma tabla de verdad. ¿Cómo pueden ser equivalentes enunciados opuestos contradictoriamente? Łukasiewicz respondió esta pregunta demostrando que aquellos enunciados que se refieren a contingentia futura están contradictoriamente opuestos, pero son, a la vez, equivalentes, es decir, portadores de un valor de verdad nuevo, el tercero.

Ad 2:

Otra causa del tardío descubrimiento de la lógica polivalente, sospecha el autor, es que no se tomó en cuenta la diferenciación aristotélica de la verdad y la falsedad. De int. 9 -el ejemplo de la batalla naval-fue, evidentemente, la fuente de inspiración del descubrimiento de la lógica polivalente, en la forma de la trivalencia. Los caps. 2-4 de An. Pr. II, en los cuales Aristóteles discute la derivación de conclusiones verdaderas a partir de conjunciones falsas de premisas, no fueron considerados desde el punto de vista de la lógica polivalente.

Es curioso que Łukasiewicz, el descubridor de la lógica polivalente, no haya prestado atención a la diferenciación aristotélica de la verdad y la falsedad.

Logik nicht weniger, als sich die nicht-euklidische Geometrie von der euklidischen unterscheide» («un sistema de lógica trivalente cuyo esbozo presenté en 1920 se diferencia de la lógica bivalente no menos de lo que la geometría no euclidiana se diferencia de la euclidiana») (Łukasiewicz 1922; 19). 
La lógica trivalente introdujo, junto a la verdad y la falsedad, por así decir, «en pie de igualdad», un tercer valor de verdad; la forma actual de la lógica polivalente ${ }^{4}$ considera, en cambio, dos valores de verdad fundamentales -como herencia de la bivalencia- y los diferencia a través de los así llamados valores de verdad derivados.

Aristóteles diferencia en su sentido -el autor quiere decir [aquí] per analogiam, por así decir, con la forma contemporánea de la lógica polivalente- la verdad sin más especificaciones, haplôs alethés, en hóle alethés y epí ti alethés; y la falsedad sin más especificaciones, haplôs pseudés, en hóle pseudés y epí ti pseudés.

En un esquema:

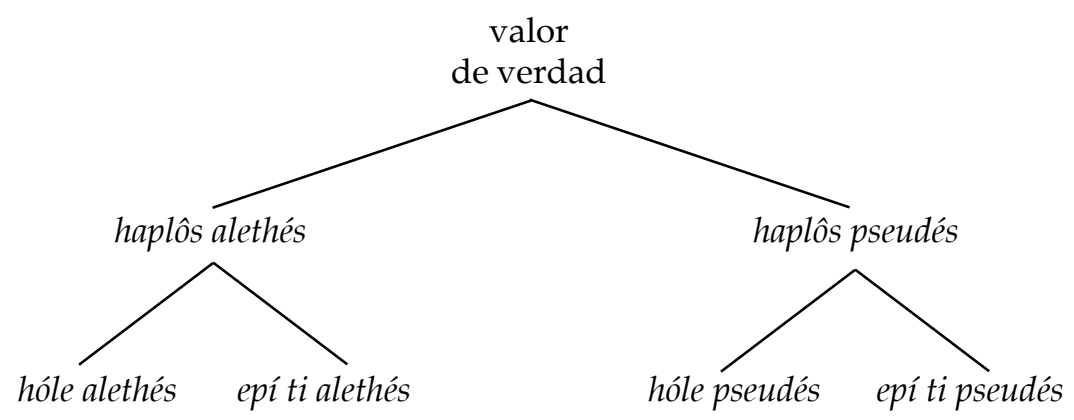

El autor sugiere, pero no desarrolla la analogía entre los valores de verdad fundamentales y derivados de la lógica polivalente, y la diferenciación aristotélica de la verdad y la falsedad sin más especificaciones.

Como es sabido, en An Pr. II, cap. 2-4, Aristóteles investiga la derivación de conclusiones verdaderas a partir de

\footnotetext{
4 Representada por A. Sinowjew y H. Wessen en «Logische Sprachregel» (1975) y por U. Blau en «Di dreiwertige Logik der Sprache» (1978).
} 
conjunciones falsas de premisas. Y advierte que las premisas que son portadoras de los valores de verdad hóle alethés/pseudés y epí ti alethés/ pseudés tienen diferentes funciones, desde el punto de vista de la inferencia silogística. Por ello, Aristóteles distingue -opina el autor- [también] en el ámbito de la falsedad -podríamos decir, per analogiam con la distinción de la lógica polivalente, que distingue valores de verdad caracterizados y no caracterizados- la falsedad universal (hóle pseudés) de la falsedad particular (epí ti pseudés). Tras constatar que de dos premisas falsas se puede derivar una conclusión verdadera (ver. An. Pr. II 2, 53b2-54a2), Aristóteles advierte que si la premisa menor es verdadera, pero la mayor falsa, de modo tal que su contraria es verdadera, entonces no se puede derivar una conclusión verdadera (adýnaton tò sympérasma alethès eînai) (ver 54a9).

Para distinguir la premisa que es falsa de modo tal que su contraria es verdadera, de la premisa que es falsa cuya contraria es también falsa, Aristóteles introduce por medio de una definición nominal el valor de verdad hóle pseudés: «Universalmente falso denomino al enunciado contrario, de modo tal que lo que no pertenece a ninguno pertenece a todos y lo que pertenece a todos no pertenece a ninguno» (54a4-6). De este modo, Aristóteles diferencia la verdad. En tal diferenciación se puede avistar unívocamente una analogía con la diferenciación de los valores de verdad no caracterizados de la lógica polivalente, que es análoga a la falsedad. Quisiéramos observar aquí que así como el ejemplo de la batalla naval ha ofrecido una fuente de inspiración para el surgimiento de la trivalencia, del mismo modo ocurre, en el caso de la tetravalencia, con la diferenciación aristotélica, en el ámbito de la falsedad sin más especificaciones, a través 
de la definición del valor de verdad «universalmente falso», que presupone, como contrapartida, la falsedad particular.

Que Aristóteles distingue la falsedad universal de la falsedad particular puede probarse, entre otras cosas, por medio del pasaje 54a19-295. Aristóteles constata que si la premisa mayor de los modos Barbara y Celarent no es universalmente sino particularmente falsa ${ }^{6}$, entonces la conclusión puede ser verdadera; si, en cambio, la premisa mayor es universalmente falsa, entonces la conclusión no puede ser verdadera.

La diferenciación de la falsedad condiciona la diferenciación de la verdad. Esta diferenciación no fue considerada, hasta donde sabemos, por los comentadores y los traductores de $A n$. Pr. Por ello, la cuestión de la diferenciación de la verdad sin otras especificaciones, tal como la aborda el autor, es de especial importancia. Especialmente, en lo que concierne a los comentarios referidos al punto, consideramos digna de mención la confrontación, justificadamente crítica, con Wieland y Ross.

Wieland afirma que «la alternativa entre completamente-falso y parcialmente-falso no encuentra en Aristóteles correspondencia en el ámbito de lo verdadero [...] si se hace abstracción del pasaje corrupto 55b9, no hay evidencia textual para la expresión "parcialmente verdadero" (epí ti alethés) ${ }^{7}$. El autor observa que el texto al que remite Wieland no está corrupto, y que, en caso de estarlo, de todos modos la aparición epí ti alethés resultaría necesaria, desde el punto de vista lógico.

An. Pr. II 2, 54a4-6.

Seguimos la traducción del autor que vierte hóle con «universal» y epí ti con «particular». Wieland 1976; fasc.1, p. 3. 
Como razón de la eliminación de epí ti alethés y, con ello, de modo indirecto, como razón de la no diferenciación de la verdad, David Ross ofrece, principalmente, el siguiente argumento: $«[\ldots]$ the distinction between a premiss which is epí ti pseudés and one which is epí ti alethés is a distinction without a difference» ${ }^{8}$.

S. Szalai, el comentador de la traducción húngara de An. $\operatorname{Pr} .{ }^{9}$, afirma que "verdadero sin otras especificaciones» $\mathrm{y}$ «parcialmente verdadero» no serían ciertas nuevas formas de la verdad, «sino interpolaciones sospechosas o variantes estilísticas de "verdadero" o de "parcialmente falso", respectivamente».

El autor se ha tomado el trabajo de demostrar, a través de una minuciosa investigación paleográfica, que el pasaje en el cual aparece epí ti alethés 55b9, passim, no está corrupto, y ha probado que epí ti alethés y epí ti pseudés son valores de verdad que pertenecen a juicios que se encuentran entre sí en relación de contradicción; identificar epí ti alethés y epí ti pseudés sería una metábasis eis állo génos; resulta, a todas luces, incorrecto identificar el valor de verdad derivado en el ámbito del concepto genérico «verdadero sin otras especificaciones» con el valor de verdad derivado en el ámbito del concepto genérico «falso sin otras especificaciones».

La «rehabilitación», por así decir, del valor de verdad epí ti alethés constituye un importante aporte de la monografía que estamos reseñando, justamente por combatir la difundida opinión que pretende eliminar ese valor de verdad.

Si Arquímedes pudo decir «dame un punto de apoyo y moveré la tierra», del mismo modo se puede derivar la de-

Ross 1949; p. 432.

Ver Aristóteles 1961; 457. 
finición de los cuatro valores de verdad a partir de la definición del valor de verdad hóle pseudés. La definición del valor de verdad hóle pseudés contiene la idea de que lo que no pertenece a ninguno pertenece a todos, y viceversa. De aquí se sigue que si un enunciado es portador del valor de verdad hóle pseudés, entonces su enunciado contrario es verdadero, es decir, si un enunciado A es portador del valor de verdad hóle pseudés, entonces su enunciado contrario, el correspondiente enunciado $\mathrm{E}$, es verdadero. Vale decir: tenemos aquí un caso especial de la relación de contrariedad. Como correlato, se sigue de aquí que si un enunciado es hóle pseudés, queda excluida la posibilidad de una predicación verdadera en la correspondiente cualidad judicativa, precisamente, en razón de la verdad del enunciado contrario. Si el enunciado A es portador del valor de verdad hóle pseudés, entonces el enunciado $\mathrm{E}$ es verdadero $\mathrm{y}$, consecuentemente, en la cualidad judicativa negativa, la verdad ha tenido lugar universaliter; por ello, en la cualidad judicativa afirmativa queda excluida la posibilidad de predicación verdadera. Si A es hóle pseudés -simbólicamente, $\mathrm{Fu}$-, entonces $\mathrm{E}$ es verdadero y U es falso, y viceversa: si $\mathrm{E}$ es $\mathrm{Fu}$, entonces $\mathrm{A}$ es verdadero $\mathrm{y}$, consecuentemente, $\mathrm{O}$ y $\mathrm{U}^{\prime}$ son falsos.

Sin embargo, y aquí se tiene el segundo aspecto de la relación de contrariedad, un enunciado universal puede ser falso también de modo tal que su contrario es también falso, es decir, su falsedad es resultado de una falacia pars pro toto: tinì hypárchontos pantì labeîn hypárchein. En ese caso, los enunciados subalternos de dicho enunciado universal falso son verdaderos. Tenemos, pues, a diferencia del caso del enunciado $\mathrm{Fu}$, una relación contradictoria del tipo «todos - algunos no - ninguno - algunos sí». La definición de estos valores de 
verdad es la siguiente: predicación universal falsa, que surge a partir de una generalización indebida.

Ahora bien, esta diferenciación de la falsedad tiene como consecuencia la diferenciación de la verdad. Más precisamente: si un enunciado es portador del valor de verdad hóle pseudés, entonces su negación es una predicación universal verdadera en la cualidad judicativa opuesta. Se obtiene así, en la cualidad judicativa opuesta, un enunciado universal que es verdadero. Si un enunciado universal es verdadero, entonces es eo ipso universalmente verdadero. Aristóteles asigna el valor de verdad hóle alethés siempre a enunciados universales; en cambio, si el enunciado es portador del valor de verdad epí ti pseudés, entonces su negación es un enunciado particular en la cualidad judicativa opuesta, cuya verdad queda limitada a la particularidad, precisamente por causa del enunciado particular verdadero, pero falsamente generalizado. Por tanto, se tiene aquí un enunciado particular verdadero, que es portador del valor de verdad epí ti alethés, $V p$.

De este modo, se puede configurar una tabla de verdad tetravalente de la negación, per analogiam con la tabla de verdad tetravalente de la negación en la lógica simbólica:

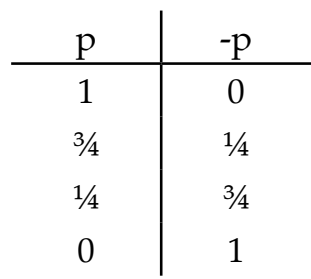

\begin{tabular}{c|c}
$\mathrm{p}$ & $-\mathrm{p}$ \\
\hline $\mathrm{Vu}$ & $\mathrm{Fu}$ \\
$\mathrm{Vp}$ & $\mathrm{Fp}$ \\
$\mathrm{Fp}$ & $\mathrm{Vp}$ \\
$\mathrm{Fu}$ & $\mathrm{Vu}$
\end{tabular}

Hemos intentado comentar los desarrollos argumentativos de la monografía reseñada, que nos parecieron interesantes desde el punto de vista del origen de la lógica polivalente, no sin introducir ocasionalmente algunas obser- 
vaciones críticas. Nos ha decepcionado que el autor, a pesar de haber puesto de relieve la contraintuitividad del tercer valor de verdad, no haya recalcado suficientemente el hecho de que la tabla de verdad trivalente de la negación implica el funcionamiento de operadores opuestos entre sí, la contravalencia y la equivalencia.

A modo de resumen, podemos anotar que en la contribución que es objeto de esta reseña, por un lado, se lleva a cabo el intento de esbozar una hipótesis referida a la causa del tardío descubrimiento de la lógica polivalente, mientras que, por otro lado, se establece una analogía que remite a la conexión entre conceptos de la moderna lógica polivalente y la terminología aristotélica. El autor comenta de modo detallado dicha analogía en la Festschrift para Hans Lenk, titulada Pragmatisches Philosophieren (pp. 99-112), un trabajo que, lamentablemente, no hemos podido considerar en esta reseña.

\section{BIBLIOGRAFÍA}

Aristóteles. (1961). Organon. 1. Katégoriák. Herméaentika. Elsö Analitika. Budapest: Akadémiai Kiadó.

Łukasiewicz, J. (1920). "Philosophische Bemerkungen zu merhwertigen System des Aussagenkalküls». En: Pearce, D. \& J. Wolenski (eds.), Logischer Rationalismus. Philosophische Schriften der Lemberg-Warschauer Schule. Frankfurt am Main: Athenäum Verlag, 1988.

. (1922). «Über den Determinismus». En: Menne, A. \& N. Öffenberger (eds.), Zur modernen Deutung der Aristotelischen Logik, vol. III. Tr. Günther Patzig. Hildesheim: Georg Olms Verlag. 
Ross, W. D. (1949). Aristotle's Prior and Posterior Analytics. A Revised Text with Introduction and Commentary. Oxford: Clarendon Press.

Wieland, W. (1976). «Probleme der Aristotelischen Theorie über Schlüsse aus falschen Prämissen». Archiv für Geschichteder Philosophie, vol. 58. 
